\title{
La educación secundaria y el distanciamiento familiar de la escuela. Un análisis longitudinal
}

\author{
Carlos Alonso Carmona \\ Universidad Pablo de Olavide \\ calonsocarmona@gmail.com
}

Recepción: 14-03-2019

Aceptación: 20-12-2019

Publicación: 01-10-2020

\section{Resumen}

La implicación familiar en la escuela tiende a decaer cuando los hijos acceden a la educación secundaria. Aquí se analizan estas transformaciones teniendo en cuenta tanto los recursos de los progenitores como la evolución de la trayectoria filial. Esta investigación se basa en el análisis longitudinal de entrevistas en profundidad y en la observación etnográfica en tres institutos de educación secundaria. El distanciamiento parental de la escuela tiene formas distintas, principalmente, en función del rendimiento de los hijos. Los recursos familiares son poco relevantes cuando los hijos obtienen buenos resultados escolares, pero adquieren importancia cuando estos empeoran.

Palabras clave: sociología de la educación; implicación parental; trayectoria escolar; clase social; análisis cualitativo

Abstract. Secondary Education and family-school detachment. A longitudinal analysis

Parental involvement tends to decline when children access to secondary education. Those transformations are analyzed taking into account both the parental resources as well as the filial trajectory. This research is based on the longitudinal analysis of in-depth interviews and ethnographic observation in three secondary schools. Parental school detachment has different ways principally according to their children's achievement. Family resources have little relevance when the child's school results are good but they gain importance when those results worsen.

Keywords: sociology of education; parental involvement; school trajectory; social class; qualitative analysis 


\author{
Sumario \\ 1. Introducción 4. Resultados \\ 2. Marco teórico 5. Conclusiones \\ 3. Metodología Referencias bibliográficas
}

\title{
1. Introducción
}

El discurso de la «implicación parental» ha ido ganando centralidad en la agenda educativa española (Consejo Escolar del Estado, 2015). Según esta perspectiva, la implicación de los progenitores en la escolaridad es un factor central para explicar el rendimiento académico del alumnado. Aunque conceptos como «implicación», «inversión», "apoyo» o "colaboración» son ambiguos (Alonso Carmona, 2014), bajo estas etiquetas suelen agruparse comportamientos como: asistir y supervisar a los hijos con las tareas escolares, mantener un contacto regular con el profesorado, transmitir unas altas expectativas a los hijos, pasar tiempo con ellos en actividades educativas o sancionarlos cuando eluden sus obligaciones académicas. El supuesto, entonces, es que la Administración y los centros escolares deben instar a las familias a intensificar estas prácticas, y con ello mejorarán los resultados de sus hijos e hijas (Llevot y Bernard, 2015).

Sobre el grado de implicación realmente existente, encontramos notables diferencias según la fuente consultada. Atendiendo a la opinión del profesorado, el apoyo de las familias a la educación formal es mucho más débil de lo que debiera, una percepción que ha permanecido inmóvil durante décadas (Vigo Arrazola et al., 2016; Marchesi, 2008; CECS, 1997). Por el contrario, los resultados de las investigaciones empíricas — que suelen consistir en encuestas a progenitores - arrojan conclusiones distintas. Muestran grandes cuotas de «implicación» en las familias (Pérez Díaz et al., 2009), que además van en aumento (Cano et al., 2019), especialmente entre las clases populares (Pérez Sánchez et al., 2014; Martín Gimeno y Bruquetas Callejo, 2014).

Pero las impresiones docentes y los resultados de las investigaciones sí que coinciden en un punto: la intervención parental en la escolaridad de los menores desciende sensiblemente durante la Educación Secundaria Obligatoria (Martín Criado y Gómez Bueno, 2017). En esta etapa, los datos también muestran una caída de la participación en las asociaciones de madres y padres y en los espacios formalizados de relación con la escuela (Parra Martínez et al., 2015). Por otro lado, las dificultades escolares se multiplican durante la educación secundaria, con especial incidencia entre el alumnado de origen popular (Martínez García, 2009). Como ambos fenómenos corren en paralelo - distanciamiento parental, por un lado, fracaso y desigualdad de resultados, por otro-, existen evidentes tentativas de establecer un vínculo causal: las familias estarían relajando sus esfuerzos "precisamente cuando es más importante». Esta idea es muy popular entre el profesorado de secundaria (Tarabini, 2015). 
Varios estudios también han vinculado el fracaso escolar en ESO con la falta de apoyo familiar a los estudios, característica de este tramo (Suárez et al., 2011; Martínez González y Álvarez Blanco, 2005). La literatura internacional ha señalado que la «implicación parental» es especialmente predictiva del rendimiento en la etapa secundaria (Eccles y Harold, 1993). En cuanto a los motivos del alejamiento familiar, veremos que las explicaciones han sido variadas. Pero en publicaciones académicas es frecuente encontrar alusiones a las creencias parentales sobre la educación (Hoover-Dempsey y Sandler, 1997). Algunas familias estarían más interesadas en los estudios, luego asumen - y mantienen con los años- su rol de sustentadoras escolares. Otras familias, por su menor interés, no asumirían ese rol o solo lo harían durante la primera infancia. Estas últimas serían, mayoritariamente, familias de clase obrera (Sheldon, 2002). Estos argumentos han sido acogidos por algunas investigaciones en nuestro país (González-Pienda y Núñez, 2005).

En objetivo del presente artículo es doble. Primero, analizar las transformaciones en la gestión familiar de la escolaridad con el paso a la etapa secundaria, matizando la naturaleza del mencionado distanciamiento. Segundo, exponer que estas transformaciones son el resultado de un proceso de interacción en el que participan los propios menores, los docentes y unos progenitores desiguales en recursos útiles. Para ello, empleamos un diseño de investigación longitudinal que combina entrevistas en profundidad a madres y padres con observación etnográfica en tres institutos de Educación Secundaria Obligatoria. El artículo se divide en cuatro partes. En el primero, realizamos una revisión teórica del tema en cuestión. En el segundo, detallamos la metodología. En el tercero, exponemos los resultados a partir del análisis de las entrevistas y las observaciones. En el cuarto y último, ofrecemos unas conclusiones y revisamos críticamente algunos hallazgos previos de la literatura.

\section{Marco teórico}

\subsection{Diferencias en apoyo escolar familiar}

Dada la importancia concedida a la implicación parental como factor de mejora educativa, muchas investigaciones se han centrado en entender por qué la inversión escolar de unos progenitores difiere de la de otros. Comprender estas diferencias sería un buen primer paso para potenciar la «implicación» de las familias (Epstein, 1996). De esta línea de investigación beben algunas iniciativas públicas que han buscado incrementar el apoyo familiar a la escuela mediante la acción de los propios agentes escolares. Sin embargo, la evaluación sistemática de este tipo de programas apunta invariablemente a la decepción. Su influencia ha sido muy discutida y sus éxitos puntuales en modificar las prácticas parentales no han traído mejoras significativas en los resultados académicos (Mattingly et al., 2002; Pomerantz et al., 2007).

Si los factores institucionales muestran un impacto tan limitado, la explicación a las diferencias debe hallarse en las propias familias. Así, se han identificado una serie de elementos que inciden en la intensidad de la inversión 
escolar, como la estructura familiar, la zona de residencia o el tiempo disponible (Hoover-Dempsey y Sandler, 1997). Pero la variable protagonista ha sido el estatus socioeconómico familiar, en parte por englobar o al menos guardar relación con el resto de características relevantes. Varias investigaciones han detectado que la implicación en lo escolar es más fuerte en los estratos sociales privilegiados, ${ }^{1}$ especialmente cuando los hijos están en secundaria (Desforges y Abouchaar, 2001; Green et al., 2007). Lareau (2000), a partir de una revisión de la literatura, identifica tres grandes mecanismos explicativos de esta diferencia:

— Las "creencias parentales»: el estatus social influiría en los valores familiares y en las aspiraciones para los hijos. Las familias de estratos sociales inferiores no se implicarían porque darían una menor importancia a la escuela y no confiarían en las capacidades académicas filiales.

- La discriminación institucional: esta teoría postula que las diferencias son creadas por las propias escuelas. El profesorado y otros agentes escolares tratarían diferencialmente a las familias en función de su posición social; las harían sentir más o menos bienvenidas y partícipes de la educación formal del menor. Con ello, impulsan que los padres de clase media continúen implicados mientras paulatinamente desincentivan a las familias obreras de hacer lo mismo (Crozier, 1999).

- Los recursos disponibles: estas explicaciones apuntan a una serie de recursos útiles para implicarse, que están desigualmente distribuidos a lo largo de la estructura social. Disponer de jornadas laborales más cortas y contar con ingresos altos y estables facilitan un seguimiento continuado de la trayectoria filial (Cooper y Stewart, 2013). Pero el recurso más estudiado ha sido el capital cultural. Aunque existen discrepancias en cuanto a la definición precisa del concepto (Mads Meier y Breen, 2016), podemos entender el capital cultural como la afinidad con el universo simbólico escolar, con los contenidos que la escuela imparte y con el lenguaje que usa para transmitirlos. Estas disposiciones permiten establecer relaciones igualitarias con el profesorado, así como entender y negociar sus demandas. Además, facilitan la supervisión o la ayuda con las obligaciones escolares de los hijos.

La primera explicación ha recibido abundantes críticas, teóricas y empíricas, por parte de investigaciones llevas a cabo en diversos países: Estados Unidos (Bennet et al., 2012), Reino Unido (Irwin y Elley, 2011), España (Martín Criado y Gómez Bueno, 2017) y Portugal (Diogo, 2010). La segunda y la tercera explicación son perfectamente compatibles, aunque las tesis de la discriminación institucional son parcialmente cuestionadas por la investigación. Es cierto que los contactos entre profesores y padres son bien distintos en fun-

1. Otros autores cuestionan parcialmente esta premisa: dependería de cómo se definiese exactamente la implicación parental. Las diferencias de clase al respecto podrían ser más cualitativas que cuantitativas (sobre este debate, véase Alonso Carmona, 2019). 
ción de la posición de clase de estos últimos (Weininger y Lareau, 2003). Sin embargo, las demandas docentes de implicación parental existen en todos los centros, independientemente del estatus social de su público (Lareau, 2000). Incluso se ha señalado que los profesores hacen más esfuerzos por acercar a las familias en institutos con baja composición social (Driessen et al., 2005). Asimismo, los programas públicos destinados a incrementar la implicación han tenido resultados exiguos, lo que pone en duda la capacidad de los agentes escolares para incidir significativamente en las prácticas familiares.

Parece entonces que los recursos parentales son el factor central para explicar las diferencias de implicación escolar, aun cuando buena parte de ellos han de entenderse en relación con las demandas de la escuela (pues es la escuela quien marca qué disposiciones familiares son útiles). En concreto, el capital cultural familiar es especialmente relevante durante la etapa secundaria. En el contexto español, los institutos son espacios más formalizados que los colegios, se multiplica el número de docentes y los contenidos a estudiar por parte de los hijos ganan complejidad. La negociación con el profesorado cobra mayor importancia: se generaliza la repetición de curso y la asignación del alumnado a distintos itinerarios -incluso en sistemas comprehensivos como el español (Castejón, 2018; Rujas, 2015). En los encuentros con los progenitores, los profesores comienzan a priorizar las cuestiones académicas frente a las actitudinales (Martín Criado et al., 2014). A ello se suma el incremento de estatus del cuerpo docente, lo que aumenta la distancia social que le separa de las familias de clases populares. ${ }^{2}$

\subsection{Trayectoria escolar de los hijos e implicación parental}

Numerosos estudios señalan que la implicación escolar que despliegan las familias varía con la edad de los hijos (Hill y Tyson, 2009). No solo en la forma e intensidad de la inversión, también en sus efectos: las prácticas familiares y los recursos que pueden movilizarse no tienen la misma importancia en todos los momentos de la trayectoria filial. Varias investigaciones muestran que sus efectos a largo plazo son mayores durante la primera infancia (Guo, 1998; Wagmiller et al., 2006; Lee, 2014), aunque algunas formas de implicación parecen ser relevantes durante la adolescencia (Hill y Tyson, 2009).

La variación en los comportamientos parentales a lo largo del tiempo está muy ligada al sentido de la trayectoria escolar filial. Los progenitores calculan su implicación atendiendo a cómo evolucionan las calificaciones de los hijos y al éxito que perciben en las medidas tomadas (Mads Meier y Breen, 2016). De ahí que, para analizar la implicación, sea necesario ponerla en relación con los vaivenes de la trayectoria filial. Pero este «ajuste» de los comportamientos parentales a los resultados filiales no es independiente de la clase social: las

2. No solo de estatus actual, también de origen: en comparación con el profesorado de infantil y primaria, el de enseñanzas medias se recluta en mucha menor proporción de entre las clases populares (Pérez Sánchez, 2000). 
familias de distinto estatus parecen reaccionar de forma diferente a las alteraciones de la trayectoria de los hijos.

En líneas generales, la literatura internacional muestra que las clases superiores redoblan su inversión cuando la trayectoria peligra (Bernardi y CebollaBoado, 2013; Quadlin, 2015) y son más proclives a usar estrategias efectivas para promocionar el rendimiento (Robinson y Harris, 2013; Cano et al., 2019) o para compensar circunstancias desfavorables de cara al aprendizaje escolar (Hsin, 2012; Bernardi, 2014; Gil-Hernández, 2019). En cambio, las formas de implicación de las clases populares y de otros sectores subalternos parecen ser menos efectivas para conseguir ventajas escolares (Lee y Bowen, 2006; Alonso Carmona, 2014). En dichos grupos, la acumulación de problemas académicos lleva con más frecuencia a un distanciamiento familiar de la escuela (Lareau, 2000; Crosnoe, 2001) y a un descenso de las expectativas (Goldenberg et al., 2001). En España, la escasez de datos empíricos ha dificultado el estudio de estos procesos, aunque algunas investigaciones encuentran pautas similares (Bernardi y Cebolla-Boado, 2014; Martín Criado y Gómez Bueno, 2017).

El impacto de las actitudes filiales sobre las prácticas parentales, así como sus efectos diferenciales en función de la clase social, ha sido extensamente estudiado a nivel cuantitativo. Esta investigación trata de profundizar en los mecanismos detrás de esta desigualdad mediante una aproximación cualitativa, en un contexto nacional poco analizado como es el español.

\section{Metodología}

Este artículo parte del análisis longitudinal de datos cualitativos recogidos para realizar una tesis doctoral actualmente en curso. La principal fuente de datos proviene de 30 entrevistas en profundidad a madres y padres residentes en la provincia de Sevilla. El primer ciclo de entrevistas fue realizado durante el primer trimestre del curso escolar 2016-2017, momento en que todos los entrevistados tenían un hijo/a recién entrado en Educación Secundaria Obligatoria. Estas entrevistas se centraron en examinar las prácticas de inversión escolar durante la Educación Primaria. La «implicación parental» es un concepto ambiguo y que puede hacer referencia a multitud de comportamientos distintos (Alonso Carmona, 2019). Se aprovechó el carácter abierto de las entrevistas en profundidad para que los sujetos definiesen su relación con la escolaridad y se centrasen en aquellas prácticas que consideraban más relevantes. Con todo, hubo una serie de temas que sí se lanzaron específicamente en todas las entrevistas: (1) posibles relaciones con el trabajo escolar del menor (vigilancia durante su realización, control de horarios, instrucción de contenidos, certificación de las tareas realizadas), (2) conversaciones cotidianas con el menor sobre la escuela, (3) expectativas académicas depositadas en el menor y (4) motivos y frecuencia de contacto con el profesorado, así como experiencias durante el mismo.

Las mismas familias fueron entrevistadas entre mayo y julio de 2018, cuando el menor estaba finalizando el segundo curso de la educación secundaria 
Tabla 1. Entrevistados por origen social, sexo y rendimiento filial

\begin{tabular}{|c|c|c|c|c|}
\hline Nivel de estudios & 0cupación & 0cupación cónyuge & $\begin{array}{l}\text { Rendimiento filial (primaria) } \\
\text { y trayectoria }(\mathrm{ES} 0)^{\star}\end{array}$ & Sexo \\
\hline Superiores (uni.) & Prof. universidad & Prof. Universidad & Alto - descendente & M \\
\hline Superiores (FP) & Propietaria gestoría & Propietario gestoría & Alto - estable & M \\
\hline Superiores (univ.) & Ingeniera & Abogado & Bajo - ascendente & M \\
\hline Superiores (univ.) & Prof. secundaria & Ama de casa & Medio - descendente & $\mathrm{H}$ \\
\hline Medios (BUP) & Propietario sector construcción & Ama de casa & Alto - estable & $\mathrm{H}$ \\
\hline Superiores (univ.) & Auxiliar de guardería & Monoparental & Medio - descendente & $M$ \\
\hline Superiores (univ.) & Psicóloga & Periodista & Medio - estable & M \\
\hline Primarios & Asistenta doméstica & Fontanero & Alto - estable & M \\
\hline Incompletos & Ama de casa & Transportista & Medio - ascendente & M \\
\hline Primarios & Asistenta doméstica & Dependiente & Bajo - estable & M \\
\hline Primarios & Cocinera & Transportista & Alto - estable & M \\
\hline Medios (BUP) & Dependiente & Administrativa & Alto - descendente & $\mathrm{H}$ \\
\hline Medios (FP) & Cocinera & Comercial & Medio - descendente & $M$ \\
\hline Superiores (univ.) & Ama de casa & Ingeniero & Alto - estable & M \\
\hline Medios (FP) & Ama de casa & Delineante & Bajo - descendente & $M^{\star \star}$ \\
\hline Medios (FP) & Ama de casa & Montador mecánico & Alto - estable & $M$ \\
\hline Primarios & Camarera & Jornalero & Medio - descendente & $M$ \\
\hline Primarios & Encuadernadora & Monoparental & Bajo - descendente & $M$ \\
\hline
\end{tabular}

* Hemos distinguidos entre rendimientos Altos (suspensos inexistentes o muy ocasionales en Primaria), Medios (suspensos recurrentes pero sin dificultades para promocionar) y Bajos (suspensos habituales con dificultades para promocionar o habiendo repetido ya un curso). La trayectoria refiere al mantenimiento, mejoramiento o empeoramiento de las calificaciones filiales en $2 .^{\circ}$ de ESO respecto a las obtenidas en Primaria.

** Ambos cónyuges estuvieron presentes en la primera entrevista. La segunda se realizó únicamente a la madre.

Fuente: elaboración propia.

(a excepción de un caso, que repitió primero). El contenido de la entrevista fue el mismo, pero circunscrito a los dos primeros años en la etapa secundaria (se introdujo, como novedad, el proceso de selección de optativas).

El contacto inicial se realizó a través de tres institutos de educación secundaria, donde se solicitó la participación voluntaria de las familias en las reuniones iniciales convocadas por los equipos directivos. La investigación se presentó como un estudio sobre los cambios de comportamiento con la llegada de la adolescencia, y sus consecuencias en la vida escolar y doméstica. Las entrevistas fueron realizadas en los hogares (a excepción de dos, realizadas en los lugares de trabajo). Las del primer ciclo tuvieron una duración de entre setenta y cinco y noventa y cinco minutos. Las del segundo ciclo, de entre cincuenta y noventa minutos. Esta diferencia se debe a la omisión de algunos temas, que no tenía sentido repetir en el segundo contacto - trayectoria escolar y laboral de los sujetos, emancipación y conformación de la familia- y a la menor extensión del periodo escolar analizado. La mayoría de participantes fueron mujeres, lo que resultó esperable: atendiendo a investigaciones previas, la relación familiar con 
la escuela es una actividad fuertemente feminizada (Weininger y Lareau, 2003; puede encontrarse un análisis exhaustivo de este fenómeno en Reay, 1998).

Procuramos que la muestra fuese variada en términos de origen social y trayectoria previa del menor. Pese al procedimiento de contacto voluntario, la muestra presenta una alta dispersión de rendimientos filiales. La diversidad de estatus socioeconómico se aseguró acudiendo a tres institutos de distinta composición social, todos de titularidad pública y situados en la provincia de Sevilla (dos de ellos en la capital y uno en un municipio cercano). El primer centro posee un reconocido prestigio en la localidad, y su público proviene mayoritariamente de las clases medias-altas. El segundo centro presenta una cierta heterogeneidad, aunque el grueso de su alumnado pertenece a las franjas estables de la clase obrera, con minorías de familias tanto de clase media como subproletarias. El tercer centro está situado en un municipio de mediano tamaño tradicionalmente agrícola. Se encuentra en la zona más humilde de la localidad y recluta a sus alumnos de entre estratos poco escolarizados de la clase obrera.

A la hora de delimitar la posición de clase de los participantes, partimos de los conceptos de capital cultural, simbólico y económico de Pierre Bourdieu, aproximándonos a ellos mediante el nivel escolar y la posición en el mercado laboral. En adelante, distinguiremos entre familias de clase obrera y familias de clases medias, tal como han procedido investigaciones similares (Lareau, 2000). Nos referimos como "de clase obrera" a familias cuyos progenitores poseen estudios primarios o medios y trabajan como asalariados en ocupaciones rutinarias descualificadas o semicualificadas. Nos referimos como "de clase media» a aquellas familias cuyos progenitores tienen estudios superiores y trabajan en ocupaciones profesionales o tienen posiciones directivas. Emplear una categorización dicotómica supone una cierta simplificación, pero también facilita la exposición de análisis comparativos, si bien durante el tratamiento de los datos se tuvo en cuenta la heterogeneidad interna de los grupos considerados y la distribución gradual de las distintas formas de capital.

Además de las entrevistas, este artículo utiliza dos fuentes adicionales de datos. Se realizaron cinco observaciones etnográficas en las reuniones de coordinación de tutores. Igualmente, se llevaron a cabo once observaciones en reuniones de tutoría entre profesores y algunos de los progenitores participantes, que pudieron ser grabadas en audio y transcritas. Las percepciones docentes y su interacción efectiva con las familias constituyen un material relevante, pues hemos visto que algunos autores les atribuyen un papel directo en la promoción o la inhibición de la inversión escolar familiar.

En las siguientes páginas analizaremos la transformación de las prácticas familiares en torno a la escuela con el paso a la etapa secundaria. Estos cambios suelen interpretarse como desinversión, como separación paulatina de los estudios filiales. Veremos que, efectivamente, la mayoría de las familias relajan el seguimiento directo de las obligaciones escolares de los hijos. Pero este distanciamiento no implica la ruptura del compromiso con los estudios filiales. Con el paso de los cursos, una serie de factores lastran a la baja la 
intensidad de la implicación parental. En algunas familias, este proceso se vive con naturalidad, y de hecho convive con una creciente ambición escolar. En otras, el distanciamiento es un proceso tenso, expuesto en las entrevistas entre múltiples ambivalencias discursivas. Pero en todas ellas las estrategias de los propios hijos juegan un papel vital. En lo fundamental, las transformaciones que constituyen nuestro objeto de estudio son producto de la cambiante relación entre demandas filiales y capacidad de agencia parental. El cuerpo docente contribuye, aunque de forma modesta, a configurar esta dinámica.

\section{Resultados}

Un factor clave que modula las estrategias familiares ante la escuela es la trayectoria acumulada de los hijos. Los éxitos y los fracasos pasados, así como la evolución reciente de las calificaciones, inciden en el tipo de prácticas que se llevan a cabo, modifican las actitudes filiales ante la escuela y van conformando un modo familiar de afrontar la escolaridad. Pero ciertos comportamientos son más intensivos en tiempo o demandan un mayor capital escolar. De ahí que, ante similares oscilaciones de la trayectoria, las prácticas familiares varían en función de sus recursos. Nuestros resultados coinciden con las teorías basadas en la desigualdad de recursos (Lareau, 2000; Bennet et al., 2012), pero añadiendo un matiz primordial: estos recursos han de ser analizados en interacción con el rendimiento previo de los hijos - pues este también es un factor que condiciona sensiblemente el abanico de estrategias parentales utilizables.

Este apartado de resultados está organizado en tres partes. En la primera, expondremos la evolución de las prácticas familiares en aquellos casos en que los menores mantienen, con el paso a la etapa secundaria, unos buenos resultados académicos. En la segunda, haremos lo propio con las familias cuyos hijos empeoran sus resultados - generalmente, tras una etapa primaria en la que ya han aparecido algunos problemas-, y su escasez de recursos dificulta gradualmente la inversión escolar. En la tercera, analizaremos a los progenitores cuyos hijos experimentan igualmente dificultades, pero pueden asumir el coste de intervenir en la escolaridad filial.

\subsection{Cuando todo va bien}

El análisis longitudinal de las entrevistas muestra una clara transformación de los discursos parentales con el cambio de etapa educativa. En general, las prácticas de implicación se hallan en constante tensión entre dos polos contradictorios pero legítimos desde el punto de vista experto: a un lado, la necesidad de vigilar los estudios filiales, al otro, el deber familiar de promocionar la autonomía infantil y adolescente. Cuando los menores acaban de finalizar la etapa primaria, ambos postulados coexisten en un equilibrio inestable. Los progenitores maniobran discursivamente para evitar ser etiquetados tanto de negligentes como de sobreprotectores (una tendencia observable en todas las fami- 
lias, pese a las enormes diferencias en el grado efectivo de inversión). Las cosas cambian durante el segundo periodo de entrevistas. La noción de autonomía va ganando peso en los discursos parentales, de modo que desplaza y estigmatiza el control continuado de la vida escolar. Este deslizamiento del punto medio en el eje discursivo obedece a una serie de factores.

La actitud de los propios hijos es uno de ellos. Cuando su rendimiento ha sido alto y estable, la vigilancia cotidiana tiende a reducirse. Ya en los últimos cursos de primaria, encontramos familias que apenas tienen contacto con las obligaciones escolares de los menores, sobre todo en lo que respecta a sus tareas para casa. Los hijos han interiorizado la cotidianeidad del trabajo escolar, organizan sus tiempos y realizan sus deberes de forma autónoma. Este proceso se intensifica durante la etapa secundaria. La única demanda parental fuerte a los hijos es la exigencia de coordinación con los ritmos de organización doméstica (calcular el tiempo necesario para que los deberes no intercedan con actividades extraescolares, rutinarias visitas a familiares, viajes u otras actividades los fines de semana, no dejar las cosas "para última hora, cuando hay que cenar y ducharse», etcétera).

Durante el segundo ciclo de entrevistas, los temas de conversación en este tipo de familias viran abundantemente a cuestiones no escolares. La buena socialización parental se presenta como la inculcación de valores morales: lo más importante es que los niños sean respetuosos, tolerantes, amables, que estén bien educados. La no intervención en el ámbito propiamente escolar es motivo de orgullo familiar, que se expresa abiertamente en las entrevistas, «el trabajo sale de ellos». Los hijos son conscientes de su posición ventajosa, y con sus estrategias contribuyen activamente a conformar este tipo de relaciones intrafamiliares. Usando sus (buenas) calificaciones como argumento, tratan de ir ganando espacio y de disuadir la - ya escasa - intervención parental. Más crecidos (en ambos sentidos), comienzan a argumentar que este tipo de vigilancia los infantiliza. El seguimiento de la escolaridad se percibe como pieza o expresión de un control familiar más amplio y, quién sabe, quizás desembarazándose de él puedan ir consiguiendo libertades en otros ámbitos:

- Parece que se quejan de que estás muy encima.

- Uy, sí, mucho, mucho. Además me dicen que no, que si yo me creo que ellas no estudian, que si yo me lo creo (...). También que como van bien... pues qué les voy a decir. Y... «pero si las notas...». Que tienen razón. Así que, bueno, la verdad que sí, que nosotros intentamos machacar siempre con ese tema. Pero es que ellas..., «si he sacado muy buenas notas», y ahora se quiere ir la mediana a dormir a casa de la amiga, $\mathrm{y}$ «si he sacado muy buenas notas»... (Mujer, clase obrera, rendimiento filial alto, trayectoria estable)

Los argumentos de las hijas minan la legitimidad del «seguir machacando» (que en esta familia y en otras similares, dista mucho de ser un control continuado). Esta tendencia se observa en familias de toda clase social cuando los menores disfrutan de trayectorias excelentes. No obstante, las condiciones de recepción de los discursos filiales son especialmente favorables cuando los 
progenitores tampoco cuentan con la capacidad objetiva de monitorizar su trabajo. Además, los menores perciben las limitaciones escolares maternas, y su agravamiento con el avance de los cursos (ello se ve muy bien en sus estrategias ante dudas puntuales: no acuden a los progenitores, si no a los amigos, hermanos mayores o a Internet). Se acrecienta entonces el hacer de la necesidad virtud: la implicación comienza a problematizarse, pues los padres no pueden y los hijos ni la piden ni la quieren.

Todo este proceso de distanciamiento no implica, como hemos señalado, una quiebra del compromiso con la escuela. Muy al contrario, estas familias - en todas las clases sociales - tienen sólidas aspiraciones universitarias y, aunque no intervengan de forma directa, hablan abundantemente con los hijos sobre temas escolares. En ambos fenómenos, los niños juegan un papel activo. Analizando los relatos de los progenitores, podemos observar que los hijos, conforme crecen, comienzan a plantearse su futuro escolar a medio plazo: las expectativas ya no son únicamente parentales, también filiales. Cuando estas últimas son altas, se transmiten a los padres, con un aumento de su motivación y la potenciación de visiones optimistas de la escolaridad filial. Este fenómeno es especialmente visible en aquellas familias con menor capital cultural ( $y$, por tanto, menor información sobre posibilidades e itinerarios ulteriores):

Hombre, que yo no sé muy bien cómo va eso, pero yo espero verla en la universidad. Ella siempre, «yo a la universidad» y «mamá, cuando yo esté en la universidad...", y yo espero verla en la universidad, mi intención es que las dos vayan a la universidad, la verdad. (...) Está muy bien encaminada. Y podría, vamos. Perfectamente. (Mujer, clase obrera, rendimiento filial alto, trayectoria estable).

Asimismo, el grado de comunicación escolar en el hogar depende de la actitud de los hijos. Aun cuando estas familias apenas intervienen en las tareas escolares cotidianas, están muy bien informadas de la vida académica filial. Dados sus buenos resultados, los menores no tienen problema en comentar con los padres la marcha cotidiana del curso. Al contrario, cuentan con el incentivo de ganar reconocimiento simbólico en el ámbito familiar, más aún cuando los hijos pueden usar sus éxitos escolares para negociar cesiones de derechos con los progenitores. De ahí que expongan abundantemente sus logros y los comparen con las dificultades de otros niños y niñas (estas familias, de hecho, suelen tener más información sobre la marcha escolar de otros alumnos).

La comunicación con el profesorado en este tipo de familias puede definirse como «ritualista». No rehúyen el contacto, pues saben que el alto rendimiento filial funciona en los centros como una patente de buena paternidad. Pero dado que obtienen abundante información de los hijos y no perciben especiales dificultades, limitan sus encuentros a las ocasiones imprescindibles (habitualmente, un poco antes o después de cada evaluación). Estas reuniones suelen ser muy cortas: los docentes comentan la buena disposición filial y los progenitores asienten orgullosos. Si bien se aprecian ciertas diferencias de clase —las familias con mayor capital cultural son más proactivas en sus intervenciones, 
comentan en mayor medida los temarios y contenidos curriculares-, estas no son tan acusadas como en los casos en que los hijos tienen problemas (que analizaremos más adelante). Además, acostumbrados a los buenos resultados y a que estos continúen pese a la escasez de seguimiento directo, estas familias pueden ignorar ciertas llamadas a la implicación por parte del profesorado. Las estrategias parentales se adaptan mucho más a las calificaciones que a otros juicios docentes:

[Hablando de una tutoría] «La niña va flojita, va a suspender aquí y aquí, mira las opiniones de otros profesores... tenéis que... tal", firmé y me dio un papel. Y luego no se parecía... Esto es como las encuestas de los políticos, luego las notas no se parecían en nada a lo que ella me había dicho. Y me citó dos semanas antes de las notas. Y al final lo aprobó todo (...), no se parecía en nada a lo que había dicho. Bueno. Con L. me pasó lo mismo, con el tutor de L., muy alarmado, «uy, no atiende, no sé qué, se despista...» y iluego? Notables, sobresalientes... o sea que... no lo estaremos haciendo tan mal. (Hombre, clase media, rendimiento filial alto, trayectoria estable)

Este hecho puede ser fuente de tensiones. Incluso con buenas trayectorias, el paso a la etapa secundaria suele estar marcado por ocasionales descensos de rendimiento en ciertas asignaturas («se ha dejado ir un poquito»). Esto coloca a los padres en una situación delicada, pues realizar pequeñas intervenciones es costoso. Falta costumbre y, a la mayor dificultad de los contenidos, se suma el hecho de que los progenitores no se han familiarizado progresivamente con ellos (por su escasez de apoyo directo en los cursos anteriores). Los menores, habituados al laissez-faire, no suelen recibir de buena gana estos novedosos intentos de control. Al hablar de las dificultades puntuales de los hijos, los discursos de estas familias - construidos en torno a la autonomía y la confian$z a$ - han de reelaborarse, con constantes modulaciones y negociaciones del sentido: no se «ayuda» sino que se «resuelven dudas», no se "castiga» sino que se «introducen limitaciones», etcétera. Pese a todo, hablamos de conflictos de escasa magnitud, en especial si los comparamos con lo que sucede en hogares donde los niños experimentan dificultades más graves. Estos casos son los que revisaremos a continuación.

\subsection{Llueve sobre mojado}

En las familias con bajo capital cultural y poca disponibilidad de tiempo, la inspección continuada de las obligaciones escolares tiende a reducirse con el paso a la ESO. Hemos visto que esto es así cuando los hijos acumulan buenos resultados, aunque algo parecido sucede cuando se dan severos descensos de rendimiento. La dinámica en juego es similar: una imbricación entre demandas filiales y escasez de recursos parentales. Pero el proceso efectivo de desenganche en estas familias es muy distinto. Distinto en los motivos, pero también en la intensidad. Los graves problemas en la ESO se dan en familias cuyos hijos ya mostraron ciertas dificultades en los últimos cursos de primaria. Durante 
el primer ciclo de entrevistas, la mayoría de estas familias ejercita una intensa implicación, generalmente a partir del control de horarios de estudio y el contacto continuado con el profesorado. Tras dos años en la educación secundaria y con el incesante deterioro de la trayectoria filial, el cuadro ha cambiado completamente.

Al igual que sus homólogos con mayor rendimiento, los menores con dificultades también instan a sus progenitores a reducir el grado de vigilancia. Por su problemática relación con la escolaridad, las tareas suponen para estos niños una actividad especialmente ardua y tediosa. En los casos más graves tratan de eludirlas completamente. En otros, intentan despacharlas cuanto antes, aun cuando ello signifique una realización parcial de las mismas (hacer solo algunos de los ejercicios asignados, estudiar superficialmente los temarios, etcétera). Así, la intervención de los progenitores suele implicar un alargamiento del trabajo y un aumento de los estándares de exigencia. Peor aún, el seguimiento parental puede dejar al descubierto sus déficits, lo que acarreará reproches, castigos y discusiones. En estos casos los progenitores relatan cómo, con el paso de los años, los hijos van aprendiendo todo tipo de tretas para eludir el control parental.

En estas familias puede hablarse abiertamente de un boicot filial a las estrategias familiares. El modo de obstaculizarlas es muy variado: ocultar las tareas estipuladas por los docentes, mentir con las fechas de los exámenes (o con las calificaciones obtenidas) o incluso extraviar oportunamente la agenda escolar. Cuando nada de esto funciona, estos menores reaccionan con negativas explícitas a los intentos parentales de monitorizar su trabajo, negativas que pueden desembocar en altercados de cierta agresividad (los hijos exhortan violentamente a las madres a que abandonen su cuarto, dan portazos, etcétera). Además, la propia naturaleza de su trabajo escolar — desordenado, escaso, irregular- dificulta aún más que los progenitores tengan un control efectivo sobre el mismo («ni él sabe dónde tiene los cuadernos»). Por si fuera poco, la comunicación cotidiana sobre la escuela es casi nula - pese a las insistencias parentales- $y$, cuando existe, se limita a anécdotas puntuales sin relación con los contenidos o aprendizajes. La escuela es un espacio incómodo para los hijos, cada vez evitan más hablar de ella, y tanto mejor si además mantienen a los padres desinformados. Una situación que se intensifica con los años, al ritmo que la vida escolar se va academizando:

Antes me contaba más cosas, ahora no me cuenta casi nada, antes... «Me ha pasado esto, tengo esto, me ha pasado tal...", cuando chico, pero ahora, ya te digo, no me entero ni de la mitad de las cosas. Antes todo, lo que había que llevar, lo que había que hacer... porque ya te digo, yo soy una madre muy responsable, hay que preparar un disfraz, comprar los materiales, si tú me lo dices a las diez de la noche, ¿¿ónde voy a comprar eso? (Mujer, clase obrera, rendimiento filial medio, trayectoria descendente)

Ante estas actitudes filiales, el control de la escolaridad adquiere progresivamente un inmenso coste emocional, su mantenimiento deteriora las relaciones 
intrafamiliares. Pero en las familias de clases populares, especialmente cuando ambos progenitores trabajan fuera del hogar, la situación es más delicada todavía. Llueve sobre mojado: a los obstáculos filiales se suman las dificultades objetivas tanto para vigilar sus obligaciones como para obtener información de las mismas. Además, tras una larga jornada laboral, dedicar el escaso tiempo de convivencia con los hijos a las cuestiones escolares - tan conflictivas en estas familias - se antoja especialmente doloroso. El seguimiento de sus obligaciones es paulatinamente más distante, lo que concede ventaja a los hijos en sus estrategias de boicot, en una dinámica que se retroalimenta. Con el tiempo, los padres van normalizando el descenso continuado de trabajo escolar en el hogar.

Un elemento paradigmático en el distanciamiento de estas familias lo constituyen los encuentros con el cuerpo docente. Durante las primeras entrevistas, estos padres son prácticamente los que más acuden a los centros. Ante los primeros problemas, la poca capacidad de apoyo directo y el progresivo ocultamiento filial de la vida escolar, convierten en imprescindibles los contactos con el profesado. Es necesario acudir al centro para obtener una información que mengua en el hogar, y puesto que no se sabe muy bien qué hacer para revertir las dificultades. Dos años después, el contacto ha disminuido drásticamente. La experiencia en estas reuniones es cada vez más negativa y los hijos hacen patente su oposición a que se produzcan estos encuentros — por la experiencia de salir perjudicados de ellos:

Pero es... negativa. Siempre dice solo cosas negativas. «No ha hecho esto, ha suspendido esto, es desordenado...", no dice nunca ni un punto positivo. Que yo de la última tutoría salí hecha polvo (...) A él no le gusta, no le gusta. Porque empieza «uy, ya estás ahí, otra vez, que esa gente nada más que hablan mal y nada más que dicen cosas malas», y ahí la verdad es que tiene razón. Porque él espera, «oye mamá, ¿y qué te han dicho?», y, claro, espera alguna cosa buena. Y me da pena. (Mujer, desclasamiento, ${ }^{3}$ rendimiento filial medio, trayectoria descendente)

Como el rendimiento filial no mejora, se alimenta una mutua desconfianza entre docentes y progenitores. Un elemento clave, que se observa con claridad en las tutorías y en las reuniones de coordinación, contribuye a esta dinámica. Antes hemos comentado que los progenitores, a la hora de calcular su inversión, priorizan las calificaciones frente a las impresiones comunicadas por los docentes. Al profesorado le ocurre algo muy similar: el grado de compromiso parental se infiere principalmente a partir del rendimiento y el comportamiento del alumno (Rujas, 2016) aunque los encuentros con las familias apunten en otro sentido. Así, muchos progenitores de alumnos bri-

3. La posición social de esta madre es singular: inmigrante, con alto capital cultural (estudios superiores), su título no es reconocido en España. En su país trabajó como profesora de secundaria, aquí combina un empleo de auxiliar de guardería con impartir clases particulares. Con dos hijos, divorciada y sin contacto con el padre, practicar una intensa implicación parental le resulta cada vez más difícil. 
llantes, cuyo seguimiento directo de la escolaridad en el hogar suele ser bajo, son considerados en los institutos como "padres que están encima». Cuando la situación es la contraria y hay problemas graves y continuados, la presencia física de la familia en el centro no sería suficiente para demostrar una verdadera implicación. Ello explica la creciente negativa de estas familias a reunirse con los profesores, pues sienten que estos les responsabilizan (de forma cada vez menos velada) de los fracasos filiales. Además, los docentes aprecian que estos padres poseen progresivamente menos información sobre la trayectoria de los hijos, dada la gradual oposición de los mismos a proporcionarla conforme los resultados empeoran. Así, por mucho que acudan, siempre serán sospechosos de haber dimitido de sus obligaciones, o de mantener con el centro relaciones puramente instrumentales. Las características familiares - socioeconómicas, étnicas - son fundamentales en este tipo de atribuciones:

Una profesora ilustra el caso de un alumno absentista [gitano, residente en una zona de la ciudad famosa por su nivel de pobreza]. Los padres han acudido al centro a disculparse, se han comprometido a vigilar estrechamente el tema, y se han mostrado muy sorprendidos y avergonzados (los profesores ríen imitando el acento y la forma de expresión del padre, con abundantes gestos corporales). La orientadora señala que el padre tiene antecedentes penales y ha estado encarcelado. Los profesores, casi al unísono, comentan: «Tienen miedo a la intervención policial», «No quieren perder las ayudas sociales», «Tienen que pasar por el aro». (Cuaderno de campo, reunión de coordinación de tutores, 2. ${ }^{\circ}$ ESO, IES-2)

La disminución de los contactos tiene consecuencias. Primero, en caso de conflictos y suspensos, deja a los progenitores a merced de las versiones de los hijos, lo que facilita aún más que estos obstaculicen la intervención parental (Martín Criado et al., 2014). Además, conforme se degradan las relaciones con los docentes, es mucho más probable que los progenitores exculpen, o al menos comprendan, las malas disposiciones filiales. Incluso pueden atribuirse directamente las dificultades a la propia actitud de un profesorado que, en las percepciones parentales, cada vez pone menos de su parte («ellos se lavan las manos»).

A la pérdida de relaciones con el cuerpo docente, se suma el distanciamiento con las familias de otros alumnos. Durante la etapa primaria, varios factores contribuyen a que haya sólidos vínculos: la socialización en el grupo de pares se realiza bajo supervisión parental (en cumpleaños, comuniones, «quedadas» en parques y placitas). Además, las actividades que proponen los colegios a las familias suelen tener un carácter más informal y estar alejadas de cuestiones académicas (representaciones teatrales, fiestas de disfraces, belenes, etcétera), frente a la participación en los institutos, centrada en la gestión, más burocratizada y exigente en sus demandas. Aun cuando estas transformaciones afectan por igual a todos los progenitores, existe evidencia de que la ruptura de los lazos con otras familias se da con mayor frecuencia en los casos de bajo rendimiento filial (Quadlin, 2015). La pérdida de este capital social significa 
el cierre de otro canal de información útil, ya que es muy habitual tratar de averiguar a través de otras madres lo que los hijos ocultan.

Pero el poco contacto con las familias de otros alumnos no significa que no existan demandas de ayuda o comparaciones con otros progenitores (parientes, amigos), si bien en muchas ocasiones los hijos de estos no acuden al mismo centro o están en otros cursos, lo que limita los beneficios de la comunicación. No obstante, estos vínculos son importantes analíticamente, pues los padres suelen enunciar sus prácticas situándolas en relación con los modos de crianza de otros progenitores cercanos. Con la llegada de la ESO, para estas familias, tales relaciones llevan invariablemente a la misma conclusión: que los padres de los buenos alumnos no proporcionan demasiado apoyo directo a los niños (como hemos visto anteriormente). Ello funciona como incentivo, o al menos como justificación, de un desenganche escolar al que inevitablemente empujan las dificultades parentales y la abierta hostilidad de los hijos.

Este desenganche es progresivo, y se expresa entre múltiples tensiones discursivas. Durante las entrevistas, las argumentaciones parentales oscilan constantemente entre la necesidad de mantener un control estricto, su deber de promocionar la autonomía adolescente y la aceptación resignada de un fracaso probable a corto plazo. Vemos que los hijos son parte activa de este distanciamiento familiar de la escuela, pero su relación de fuerzas con los progenitores no es la misma en todas las familias. Cuando los padres cuentan con mejores recursos, la posibilidad de éxito de sus estrategias desciende. Esto es lo que veremos a continuación.

\subsection{Padres al rescate}

En los casos de hijos con altos rendimientos, apenas se perciben diferencias en la implicación parental. La confianza en las capacidades filiales lleva a un distanciamiento paulatino de su trabajo en el hogar, así como a limitados - y protocolarios - encuentros con el profesorado. Todo cambia radicalmente cuando la trayectoria es problemática. Las familias se ven obligadas a mantener su intervención, y el diferencial de recursos disponibles (culturales, económicos, tiempo) se hace especialmente visible.

Ya hemos comentado que cuando la relación de los hijos con el ámbito escolar es tensa, tratan de que los padres se inmiscuyan lo mínimo posible. Este fenómeno puede observarse igualmente en familias de estratos sociales superiores, pero aquí los hijos no lo tienen tan fácil. Estas familias también se quejan sistemáticamente de que los niños cuentan muy poco de lo que les pasa en la escuela. Pero la afinidad de los progenitores con el universo escolar, su mayor conocimiento de los contenidos y su capacidad para evaluar el grado de cumplimiento de las obligaciones filiales — muchas veces, al margen de la información docente - limitan abrumadoramente la capacidad de los menores para esquivar el control directo. Los niños son conscientes de las capacidades parentales, lo que les lleva a afinar sus estrategias (por ejemplo, priorizando al progenitor más indulgente, habitualmente la madre), pero eso tampoco es ninguna garantía: 
Es muy inquieto, todo lo quiere hacer muy rápido (...), «ya me lo sé», le preguntamos, no se lo sabe, ¿para qué corres si sabes que te voy a preguntar? Échale un poquito más de tiempo, a ver si te lo sabes. «Pregúntame, que me vas a preguntar cuatro o cinco cosas que me sé, y... y ya me dedico a jugar, a la tablet, o lo que sea...». Entonces el tema es ese, entonces a la hora de preguntar prefiere que le pregunte mi mujer a que le pregunte yo. Porque como soy del gremio [es profesor de instituto], sé por donde preguntar para saber si te lo sabes o no te lo sabes. (Hombre, clase media, rendimiento filial medio, trayectoria descendente)

A la mayor sintonía con el contenido curricular, hay que añadir que estas familias disponen de más tiempo. Es habitual que sus jornadas laborales sean más cortas y flexibles ${ }^{4}$ y que contraten servicio doméstico. Los intentos filiales de distanciar a la familia de sus compromisos escolares suelen caer en saco roto. Ello puede tener consecuencias a largo plazo, al acabar asumiendo que el trabajo escolar es ineludible: los progenitores siempre irán un paso por delante de ellos. Por otro lado, estos padres cuentan con la capacidad de externalizar el apoyo escolar, mediante clases particulares, academias o - en casos muy graves- internados. En ocasiones, el hipotético recurso a estas instituciones funciona como amenaza para minar la resistencia filial. Los hijos aceptan el mal menor del apoyo parental antes que asumir consecuencias más drásticas.

En su relación con los docentes, las prácticas de estas familias son el caso paradigmático de implicación como modificación (Reay, 1998). Incluso en el centro de clases medias, los docentes suelen atribuir el bajo rendimiento a una falta de compromiso familiar (aun cuando esta se impute más a situaciones familiares puntuales que a una mala voluntad parental). Pero estas impresiones pueden ser pugnadas por los progenitores durante las reuniones de tutoría, merced, primero, a su mayor capital lingüístico y simbólico. Segundo, a su mayor información sobre la trayectoria — pues en comparación con familias de clases populares, son mucho más capaces de romper las barreras a la implicación que construyen los hijos. Tercero, a su capacidad de prestar apoyo pedagógico directo (actuando como enseñantes en el hogar), lo que implica un gran conocimiento de los contenidos a estudiar:

Padre: Sí, ese tema... estuve yo ayer por la tarde, estúdiatelo, venga, vuelve a estudiártelo porque te lo sabes... pero muy por encima y a veces te confundes. Venga, vente otra vez... y así. Y bueno después le pegas un repasito y todo lo demás. El tema de... los tipos de textos narrativos, lo llevaba un poco más flojito, lo demás bien, pero eso había que repasar. Claro, como estoy yo, sí puedo un poquito encauzar la situación.

Profesora: Perfecto... pues... ya me quedo yo más tranquila. (Tutoría, hombre, clase media, rendimiento filial medio, trayectoria descendente)

4. Una madre participante en esta investigación llegó a pedir una reducción de jornada, específicamente, para tener más tiempo para ayudar a su hija con sus estudios. 
Otra característica que distingue a estas familias es su capacidad para desentrañar los métodos de evaluación docente. En las tutorías, exigen mucha más información sobre en qué fallan exactamente sus hijos: quieren ver los exámenes, conocer los criterios de puntuación, la jerarquía de contenidos curriculares, etcétera. Con esta información, y gracias a su alto capital cultural, pueden hacer más eficiente su apoyo en el hogar (a la hora de ayudar con las tareas o de tomar la lección). No solo eso, también pueden disputar los juicios docentes, incluidas las calificaciones. Mediante la instrucción directa de contenidos en el hogar, van conformando su propia evaluación de las capacidades filiales. Y si ellas no coinciden con lo que muestran las notas, lo hacen saber. Aunque estas estrategias son arriesgadas — pueden provocar reacciones defensivas en los docentes-, tienen importantes efectos. El profesorado es consciente de que la familia mira con lupa las calificaciones, y esto lo obliga a asignarlas con sumo cuidado y a intensificar su atención a estos niños (Hassrick y Schneider, 2009):

Profesora: Yo soy la primera sorprendida con los suspensos, pero me sorprende más aún porque pensaba que ustedes, de alguna manera, lo intuían...

Padre: Mi hija en esta evaluación, se lo digo sinceramente, mi hija se ha portado, y con esto no pongo en tela de juicio el resultado de esos exámenes, o la calificación de esos exámenes (Profesora: No, está claro), pero le puedo asegurar con total honestidad que mi hija se ha esforzado. Entonces, yo quiero ver esos exámenes, porque no entiendo nada, porque no quiero señalar a nadie, pero a ver si el problema está en... porque en casa llega, se pone con sus deberes, y está un rato importante, todos los días. (Tutoría, pareja de clase media, rendimiento filial bajo, trayectoria ascendente)

Aunque la inversión escolar de estas familias sea más fácil que en otras de menor estatus, esto no quiere decir que se viva placenteramente. Las relaciones con el profesorado, ventajosas pero potencialmente conflictivas, pesan como una losa en las entrevistas: muchas de estas intervenciones no se ven legítimas (pero "por los hijos, lo que sea»). Además, al igual que sus homólogas de clases populares, comparan sus prácticas con otras familias, y perciben acusaciones de ser sobreprotectoras, facilitadas por el hecho de que sus parientes y amigos cuyos hijos tienen buen rendimiento muestran una menor inversión escolar. Por eso la continuidad del seguimiento conforme los cursos avanzan obliga a cada vez más elaboradas justificaciones discursivas. De ahí, una ambivalencia que se repite durante las entrevistas: los constantes relatos sobre el apoyo a la escolaridad filial se intercalan con la metáfora del ir soltando, de ayudar, pero intentar hacerlo cada vez menos.

\section{Conclusiones}

Las investigaciones sobre la intensidad y las formas de la implicación parental en la escuela han girado frecuentemente alrededor de la clase social familiar. Pero un análisis en profundidad del fenómeno requiere analizar la interacción entre recursos de clase y desarrollo de la trayectoria filial. A partir del análisis de las 
entrevistas, parece que cuando los menores van consolidando una trayectoria exitosa, el seguimiento parental directo decae y el contacto con los docentes es escaso y poco problemático. En estos casos, el estatus social familiar es poco relevante para entender la relación familiar con la escolaridad. Los recursos parentales se movilizan fundamentalmente en casos de conflicto, tanto con el profesorado como con los hijos. De ahí que pierdan valor cuando la interacción con unos y otros es apacible.

Las diferencias de clase se agudizan cuando los resultados empeoran. La creciente complejidad de los contenidos escolares, la distancia social respecto al profesorado y la oposición filial suponen enormes obstáculos para las familias de clases populares, que se ven empujadas a un progresivo distanciamiento. Las familias de estratos superiores tienen mayor capacidad para superar estas barreras y mantener su inversión con el paso de los cursos. Estos procesos tienden a retroalimentarse en interacción con los hijos, por lo que las diferencias aumentan a lo largo del tiempo. En familias de clases populares, la dificultad para mantener un seguimiento facilita que las resistencias de los hijos tengan éxito, lo que dificulta aún más el control escolar. En familias de clase media y superior, los progenitores pueden bloquear los comportamientos filiales más refractarios cuando empiezan a germinar, para impedir que estos se desarrollen y agraven. En los grupos privilegiados, la implicación parental actuaría como un «mecanismo de ventaja compensatoria» (Bernardi, 2014), que, a su vez, tendría efectos acumulativos a largo plazo (DiPrete y Eirich, 2006).

No puede sostenerse que las diferencias de clase en apoyo escolar sean el producto de una desigual valoración de la educación, al menos en la etapa escolar considerada (transición entre la educación primaria y secundaria). Primero, porque solo son perceptibles en casos de bajos rendimientos. Segundo, porque el desenganche de un sector de familias de clases populares se plantea en las entrevistas como un proceso tenso, obligado por las circunstancias y que choca con los deseos parentales. También es matizable que las propias escuelas provoquen estas diferencias. El profesorado juzga diferencialmente a las familias, pero lo hace principalmente a partir del rendimiento del hijo (y de su comportamiento, que suele deteriorarse a la par) y de la información que los progenitores demuestran poseer sobre su trayectoria.

Esto último explica que, al empeorar las calificaciones, la relación con los docentes se degrade con más intensidad en familias con menos recursos útiles (y, por tanto, con más dificultades para mantenerse al corriente de la carrera escolar filial). La influencia del estatus social familiar sobre las relaciones con el profesorado se ha interpretado habitualmente en términos de mayor o menor proximidad social y cultural (Weininger y Lareau, 2003). Pero también son de vital importancia las distintas prácticas de intervención que la familia puede emplear en el hogar. Estas suponen un acceso diferencial a información sobre la cotidianeidad escolar de los menores, y dicha información es simbólicamente recompensada en las interacciones con el profesorado.

Esta investigación pone de relevancia que para entender las prácticas parentales hay que analizar las estrategias y los rendimientos filiales. La literatura 
sobre la cuestión ha contemplado esta posibilidad (Mads Meier y Breen, 2016; Bakker y Denessen, 2007), pero lo ha hecho con frecuencia desde la lógica de la adaptación. Los progenitores calcularían la intensidad de su inversión en función de los resultados obtenidos: bien porque intensifiquen su implicación ante malos resultados, bien porque la abandonen cuando dicha implicación no se ve necesaria o no da sus frutos. El análisis de los discursos parentales refleja que los hijos configuran la relación con la escuela de forma activa, boicoteando o incentivando ciertas prácticas de inversión. La «implicación parental» no es una decisión de los progenitores, sino un proceso de relaciones entre padres e hijos.

A partir de los presentes hallazgos, podrían reinterpretarse ciertos estudios sobre implicación familiar. Las investigaciones en educación secundaria han detectado que los alumnos escolarmente exitosos provienen de familias con altas expectativas, donde existe abundante comunicación sobre la escuela, pero poca intervención directa en los estudios (Hill y Tyson, 2009). Nuestros resultados apuntan en el mismo sentido. Pero más que postular esta configuración de prácticas como "potenciadora» del rendimiento, concluimos que es el producto de un proceso de interacción entre padres e hijos, favorecido por el buen rendimiento de los últimos.

En cuanto a las limitaciones del estudio, hemos de destacar que el tiempo transcurrido entre ambos momentos de contacto es relativamente corto y que solo contamos con dos olas (limitaciones ambas que son el resultado de constricciones ligadas a la financiación). Asimismo, no contamos con datos directos de las estrategias y preferencias de los hijos, sino que nos hemos aproximado a ellas a partir de los relatos de progenitores y profesores. La implicación parental es un fenómeno complejo, y los datos disponibles no nos permiten mantener bajo control todas las variables en juego. Por todo ello, esta investigación no puede establecer relaciones precisas de causalidad (Hernán y Robins, 2019), pero sí apuntar ciertas tendencias que podrían ser tenidas en cuenta en posteriores estudios. Futuras investigaciones deberían profundizar en los procesos de desenganche escolar familiar con datos longitudinales más robustos, y analizar con mayor precisión el papel de los hijos en la configuración de dichos procesos.

\section{Referencias bibliográficas}

Alonso Carmona, Carlos (2014). «Familia, escuela y clase social: sobre los efectos perversos de la implicación familiar». Revista de la Asociación de Sociología de la Educación, 7 (2), 395-409.

- (2019). «Implicación parental, capital cultural y trayectoria filial. Prácticas y estrategias discursivas». Revista Internacional de Sociología, 77 (3), e132. <https://doi.org/10.3989/ris.2019.77.3.18.024>

BAKker, Joep y Denessen, Eddie (2007). «The concept of parent involvement. Some theoretical and empirical considerations». International Journal about Parents in Education, 1 (0), 188-199.

Bennet, Pamela R.; Lutz, Amy y Jayaram, Lakshmi (2012). «Beyond the schoolyard: The role of parenting logics, financial resources and social institutions in 
the social class gap in structured activity participation». Sociology of Education, 85 (2), 131-157.

<https://doi.org/10.1177/0038040711431585>

Bernardi, Fabrizio (2014). «Compensatory advantage as a mechanism of educational inequality: a regression discontinuity based on month of birth». Sociology of Education, 87 (2), 74-88. <https://doi.org/10.1177/0038040714524258>

Bernardi, Fabrizio y Cebolla-Boado, Héctor (2013). «Previous school results and social background: compensation and imperfect information in educational transitions». European Sociological Review, 30 (2), 207-217. <https://doi.org/10.1093/esr/jct029>

- (2014). "Clase social de origen y rendimiento escolar como predictores de las trayectorias educativas». Revista Española de Investigaciones Sociológicas, 146 (1), 3-21. $<$ https://doi.org/10.5477/cis/reis.146.3>

Cano, Tomás; Perales, Francisco y Baxter, Janeen (2019). «A Matter of Time: Father Involvement and Child Cognitive Outcomes». Journal of Marriage and Families, 81 (1), 164-184. $<$ https://doi.org/10.1111/jomf.12532>

CASTEjón, Alba (2018). Expectativas docentes, agrupamiento del alumnado y segregación escolar: una etnografía en entornos de alta complejidad en Cataluña (tesis doctoral). Barcelona: Universitat Autònoma de Barcelona.

CECS (1997). La educación a debate. Encuesta entre padres y profesores. Madrid: Fundación Encuentro.

Consejo Escolar del Estado (2015). La participación de las familias en la educación escolar. Madrid: Ministerio de Educación, Cultura y Deporte.

Cooper, Kerris y Stewart, Kitty (2013). Does money affect children outcomes? A systematic review. York: Joseph Rowntree Foundation.

Crosnoe, Robert (2001). «Academic orientation and parental involvement in education during high school». Sociology of Education, 74, 3, 210-230. <https://doi.org/10.2307/2673275>

Crozier, Gill (1999). "Is it a case of "We know when we're not wanted"? The parents' perspective on parent-teacher roles and relationships». Educational Research, 41 (3), 315-328. <https://doi.org/10.1080/0013188990410306>

Desforges, Charles y AвouchaAr, Alberto (2003). The impact of parental involvement, parental support and family education on pupil achievement and adjustment: a literature review. Nottingham: DfES publications.

Diogo, Ana Matías (2010). «Do involvimento dos pais ao sucesso escolar dos filhos: mitos, críticas e evidências». Revista Luso-Brasileira da Educaçao, 1, 71-96.

Diprete, Thomas A. y Eirich, Gregory M. (2006). "Cumulative advantage as a mechanism for inequality: a review of theoretical and empirical developments». Annu. Rev. Sociol. 32, 271-297. <https://doi.org/10.1146/annurev.soc.32.061604.123127>

Driessen, Geert; Smit, Frederik y SleEgers, Peter (2005). «Parental Involvement and Educational Achievement». British Educational Research Journal, 31 (4), 509-532. <https://doi.org/10.1080/01411920500148713>

ECClEs, Jacquelynne y Harold, Rena (1993). «Parent-school involvement during the early adolescent years». Teachers College Record, 94, 568-587. 
Epstein, Joyce (1996). «Perspectives and previews on research and policy for school, family and community partnerships». En: Booth, A. y Dunn, J. F. (eds.). Family-school links: How do they affect educational outcomes? Mahwah: Lawrence Erlbaum.

Gil-Hernández, Carlos J. (2019). «Do Well-off Families Compensate for Low Cognitive Ability? Evidence on Social Inequality in Early Schooling from a Twin Study». Sociology of Education, 92 (2), 150-175. <https://doi.org/10.1177/0038040719830698>

Goldenberg, Claude; Gallimore, Ronald; Reese, Leslie y Garnier, Helen (2001). "Cause or Effect?» A longitudinal study of immigrant latino parents' aspirations and expectations, and their children's school performance». American Educational Research Journal, 38 (3), 547-582.

González-Pienda, Julio Antonio y Núñez, José Carlos (2005). «La implicación de los padres y su incidencia en el rendimiento de los hijos». Revista de Psicología y Educación, 1 (1), 115-134.

Green, Christa L. et al. (2007). «Parents' motivation for involvement in children's education: an empirical test of a theoretical model of parental involvement». Journal of Educational Psychology, 99 (3), 532-544. <https://doi.org/10.1037/0022-0663.99.3.532>

Guo, Guang (1998) «The timing of the influences of cumulative poverty on children's cognitive ability and achievement». Social forces, 77 (1), 257-287. <https://doi.org/10.2307/3006017 >

HAssRick, Elizabeth M. y SchneIder, Barbara (2009). «Parent Surveillance in Schools: A question of social class». American Journal of Education, 115 (2), 195-225. <https://doi.org/10.1086/595665>

Hernán, Miguel y Robins, Jamie M. (2019). Causal inference: What if. Boca Raton: Chapman \& Hall/CRC.

Hill, Nancy y Tyson, Diana F. (2009). «Parental involvement in middle school: a meta-analytic assessment of the strategies that promote achievement». Developmental Pscyhology, 45 (3), 740-763. <https://doi.org/10.1037/a0015362>

Hoover-Dempsey, Kathleen V. y Sandler, Howard M. (1997). «Why do parents become involved in their children education?». Review of Educational Research, 67 (1), 3-42. $<$ https://doi.org/10.2307/1170618>

Hsin, Amy (2012). «Is biology destiny? Birth weight and differential parental treatment». Demography, 49 (4), 1.385-1.405. $<$ https://doi.org/10.1007/s13524-012-0123-y>

IRWIN, Sarah y ElleY, Sharon (2011). «Concerted cultivation? Parenting values, education and class diversity». Sociology, 45 (3), 480-495. <https://doi.org/10.1177/0038038511399618>

Lareau, Annette (2000). Home advantage: Social class and parental intervention in elementary education. Rowman \& Littlefield Publishers.

LEE, Dohoon (2014). «Age trajectories of poverty during childhood and high school graduation». Sociological Science, 1, 344-365. $<$ https://doi.org/10.15195/v1.a21>

LeE, Jung-Sook y Bowen, Natasha K. (2006). «Parent involvement, cultural capital and the achievement gap among elementary school children». American Educational Research Journal, 43 (2), 193-218. <https://doi.org/10.3102/00028312043002193> 
Llevot, Nuria y Bernard, O. (2015). «La participación de las familias en la escuela: factores clave». Revista de la Asociación de Sociología de la Educación, 8 (1), 57-60.

Mads Meier, Jaeger y Breen, Richard (2016). "A dynamic model of cultural reproduction». American Journal of Sociology, 121 (4), 1.079-1.115.

MarCHesi, Álvaro (2008). «Las emociones y los valores del profesorado». Ábaco: Revista de Cultura y Ciencias Sociales, 55, 69-78. <https://doi.org/10.2139/ssrn.2208961>

Martín Criado, Enrique y Gómez Bueno, Carmuca (2017). «El mito de la dimisión parental. Implicación familiar, desigualdad social y éxito escolar». Cuadernos de Relaciones Laborales, 35 (2), 305-325. <https://doi.org/10.5209/crla.56777>

Martín Criado, Enrique; Río Ruiz, Manuel Ángel y Carvajal Soria, Pilar (2014). «Prácticas de socialización y relaciones con la escolaridad de las familias más alejadas de la norma escolar». Revista de la Asociación de Sociología de la Educación, 7 (2), 429-448.

Martín Gimeno, Rubén y Bruquetas Callejo, Carlos (2014). «La evolución de la importancia del capital escolar en la clase obrera». Revista de la Asociación de Sociología de la Educación, 7 (2), 373-394.

Martínez García, José Saturnino (2009). «Fracaso escolar, PISA y la difícil ESO». Revista de la Asociación de Sociología de la Educación, 2 (1), 56-85.

Martínez GonzÁlez, Raquel-Amaya y Álvarez Blanco, Lucía (2005). «Fracaso escolar y abandono temprano en Educación Secundaria Obligatoria: implicación de la familia y los centros escolares». Aula Abierta, 95, 127-146.

Mattingly, Doreen; Prislin, Radmila; McKenzie, Thomas L.; Rodríguez, James y Kayzar, Benda (2002). "Evaluating evaluations: The case of parental involvement programs». Review of Educational Research, 72 (4), 549-576. <https://doi.org/10.3102/00346543072004549>

Parra Martínez, Joaquín; García Sanz, María de la Paz, Gomariz Vicente, María de los Ángeles y Hernández Prados, María de los Ángeles (2015). «La participación de las familias en los Consejos Escolares de los centros». En: Consejo Escolar Del EstAdo (ed.). La participación de las familias en la educación escolar. Madrid: Ministerio de Educación, Cultura y Deporte.

Pérez Díaz, Víctor; Rodríguez, Juan Carlos y Fernández, Juan Jesús (2009). Educación y familia: los padres ante la educación general de sus hijos en España. Madrid: Fundación Cajas de Ahorros.

PÉrez SÁnchez, Carmen Nieves (2000). «La escuela frente a las desigualdades sociales. Apuntes sociológicos sobre el pensamiento docente». Revista Iberoamericana de Educación, 23, 189-212.

Pérez Sánchez, Carmen Nieves; Betancort, Moisés y Cabrera, Leopoldo (2014). "Inversión pedagógica y éxito escolar del alumnado de clase obrera». Revista de la Asociación de Sociología de la Educación, 7 (2), 410-428.

Pomerantz, Eva M.; Moorman, Elizabeth A. y Litwack, Scott D. (2007). «The how, whom and why of parents' involvement in children academic lives: more is not always better». Review of Educational Research, 77 (3), 373-410. <https://doi.org/10.3102/003465430305567>

Quadlin, Natasha (2015). "When children affect parents: Children's academic performance and parental investment». Social Science Research, 52, 671-685. <https://doi.org/10.1016/j.ssresearch.2014.10.007> 
REAY, Diane (1998). Class Work: Mother's involvement in their children's primary schooling. Londres: UCL Press.

Robinson, Keith y Harris, Angel L. (2013). «Racial and social class differences in how parents respond to inadequate achievement: consequences for children's future achievement». Social Science Quarterly, 94 (5), 1.346-1.371. <https://doi.org/10.1111/ssqu.12007>

Rujas, Javier (2015). Sociología del «fracaso escolan» en España. Construcción y gestión de un problema social (tesis doctoral). Madrid: Universidad Complutense de Madrid.

- (2016). "Cómo juzga la escuela a las familias». Revista de la Asociación de Sociología de la Educación, 9 (3), 385-396.

Sheldon, Steven B. (2002). "Parents' social networks and beliefs as predictors of parent involvement». The Elementary School Journal, 102 (4), 301-316. <https://doi.org/10.3102/0002831208316205>

Suárez, Natalia; Tuero-Herrer, Ellián; Bernardo, Ana; Fernández, Estrella; Cerezo, Rebeca; González-Pienda, Julio; Rosario, Pedro y Núñez, José Carlos (2011). «El fracaso escolar en la educación secundaria: análisis del papel de la implicación familiar». Revista de Formación del Profesorado e Investigación Educativa, 24, 49-64.

TARABini, Aina (2015). "La meritocracia en la mente del profesorado: un análisis de los discursos docentes en relación al éxito, fracaso y abandono escolar». Revista de la Asociación de Sociología de la Educación, 8 (3), 349-360.

Vigo Arrazola, Begoña; Dieste, Belén y Julve, Carmen (2016). «Voces sobre participación de las familias en la escuela y éxito escolar». Revista de la Asociación de Sociología de la Educación, 9 (3), 320-333.

Wagmiller, Robert L.; Lennon, Mary Clare; Kuang, Li; Alberti, Philip M. y Aber, J. Lawrence (2006). "The dynamics of economic disadvantage and children's life chances». American Sociological Review, 71 (5), 847-866. <https://doi.org/10.1177/000312240607100507>

Weininger, Elliot B. y Lareau, Annette (2003). «Translating Bourdieu into American context: The question of social class and family-school relationships». Poetics, 31 (5-6), 375-402. <https://doi.org/10.1016/s0304-422x(03)00034-2> 